Iustitia Socialis. Revista Arbitrada de Ciencias Jurídicas.

Año IV. Vol. IV. N 1 . Edición Especial 2019

Hecho el depósito de Ley: FA2016000064 ISSN: 2542-3371

FUNDACIÓN KOINONIA (F.K). Santa Ana de Coro, Venezuela

María Yokir Reyna Zambrano

http://dx.doi.org/10.35381/racji.v4i1.546

\title{
Destitución de jueces por error inexcusable y su afectación a los derechos - garantías constitucionales
}

\section{Dismissal of judges for unexcusable error and their impact on rights - constitutional guarantees}

\author{
María Yokir Reyna Zambrano \\ yokirreyna@hotmail.com \\ Universidad San Gregorio de Portoviejo, Manabí \\ Ecuador \\ https://orcid.org/0000-0003-0524-0399
}

Recibido: 30 de octubre de 2019

Aprobado: 03 de diciembre de 2019

\section{RESUMEN}

El artículo es un avance de un proyecto de investigación institucional, el cual tiene por objetivo analizar la destitución administrativa de jueces en el Estado Ecuatoriano por error inexcusable y su afectación a los derechos y garantías constitucionales, período 2011-2017. Se empleó una metodología documental - bibliográfica, lo cual permite presentar un adelanto analítico de un trabajo investigativo en desarrollo, teniéndose como muestra poblacional; documentos, leyes, investigaciones, inherentes al tema planteado. Se concluye que las afectaciones son numerosas, tales como las realizadas a los ciudadanos al acudir a la justica, al Estado de Derecho, a la Carrera Judicial y a los derechos del juez como funcionario público.

Descriptores: Constitución; derecho administrativo; derecho público; derecho constitucional.

\section{ABSTRACT}

The article is an advance of an institutional research project, which aims to analyze the administrative dismissal of judges in the Ecuadorian State for inexcusable error and its impact on constitutional rights and guarantees, 2011-2017 period, using a documentary methodology - bibliographic, which allows to present an analytical advance of a research work in development, having as a population sample, documents, laws, research, inherent to the topic raised. It is concluded that the affectations are numerous, such as those made to citizens when they go to justice, the State of Rights, the Judicial Career and the rights of the judge as a public official.

Descriptors: Constitutions; administrative law; public law; constitutional law. 


\section{INTRODUCCIÓN}

El 13 de julio de 2011 mediante reformas al Sistema de Justicia, se incorporó en el Código Orgánico de la Función Judicial del Ecuador (en adelante COFJ), como atribución del Consejo de la Judicatura, la potestad de sancionar con destitución al juez que en las causas a su cargo hubiere incurrido con dolo, manifiesta negligencia o error inexcusable (Asamblea Nacional del Ecuador, 2011), la problemática a esta causal de destitución ronda en virtud a la ausencia de contenido que el legislador olvidó darle. Consecuentemente, sin señalar el alcance claro de la conducta infractora, dicha causal se ha convertido en un continente sin contenido, sin una definición objetiva, ni elementos esenciales, transformándola en lo que la doctrina conoce como conceptos jurídicos indeterminados, esto es igual a la vaguedad y ambigüedad.

De esta manera, se dejó en manos del órgano administrativo el control de actos jurisdiccionales, estableciendo diversos criterios o parámetros para la ejecución de dicha potestad no atribuida, lo que conllevaría al riesgo de la vulneración del principio de independencia judicial y de seguridad jurídica. En un Estado Constitucional este conjunto de garantías destinadas a asegurar la efectividad del sistema judicial, el respeto a las partes del proceso, a las instituciones políticas y a proteger la libertad de los ciudadanos, constituye un núcleo esencial del sistema de justicia en el que se articula cualquier Estado constitucional.

Considerando que las garantías que rodean la función jurisdiccional no pueden ser transgredidas por la aplicación de figuras que generen interferencias en la administración de justicia a partir de potestades sancionatorias atribuidas al órgano administrativo, como es el caso del error inexcusable en el sistema disciplinario judicial ecuatoriano, es fundamental conocer cuáles son los problemas conceptuales, doctrinarios y jurisprudenciales bajo los cuáles los jueces, el sistema y la ciudadanía ecuatoriana se están viendo afectados, por consiguiente se podrán saber los elementos que están en juego en un Estado de Derecho.

El estudio planteado tiene una connotación en el ordenamiento jurídico y la actualidad del Ecuador, debido a que el Pleno del Consejo de Participación Ciudadana y Control Social Transitorio, conformado en el año 2018, reconoció que la 
figura jurídica del error inexcusable como causal de destitución, trajo como consecuencia un sub sistema de control sancionatorio que transgredió derechos de los servidores judiciales (Pásara, 2015), siendo así ¿Hasta qué punto el mismo Estado ha causado un perjuicio para consigo mismo? ¿Es necesario eliminar del ordenamiento ecuatoriano dicha figura de destitución, o en su defecto se le debe dar una definición a este término ambiguo?

En concordancia a lo previsto, el artículo se presenta como un adelanto del primer objetivo específico del proyecto de investigación titulado: "La Destitución Administrativa de Jueces en el Estado Ecuatoriano por error inexcusable y su afectación a los derechos y garantías constitucionales, período 2011-2017", aprobado por el Consejo Universitario de la Universidad San Gregorio de Portoviejo, por lo cual tiene como objetivo general analizar la destitución administrativa de jueces en el Estado Ecuatoriano por error inexcusable y su afectación a los derechos y garantías constitucionales, período 2011-2017.

\section{MÉTODO}

Para el desarrollo de la investigación se utilizará el método lógico deductivo, pues a partir de los derechos, principios y garantías vulnerados por la aplicación de la figura (error inexcusable) se conocerá el grado de afectación que se tiene hacia el Estado, la ciudadanía, la Carrera Judicial y no menos importante, a los jueces. Existen algunas limitaciones que podrían surgir en la investigación, como localizar a las personas cuyos casos fueron los más emblemáticos a nivel nacional, así como también la posibilidad de la existencia del sesgo de información en las entrevistas, adicionalmente los aspectos ideológicos que pueden derivar a una opinión poco imparcial y el tiempo que el Consejo de la Judicatura demore en responder las peticiones que se efectúen para obtener la información requerida.

Además de ello, la metodología tendrá un enfoque mixto, dentro de la parte cualitativa se hizo uso de las entrevistas a profundidad, actualmente se están aún llevando a cabo con especialistas del área administrativa, constitucionalistas, y expertos del derecho disciplinario, y con ex jueces, destituidos durante el período a ser objeto de análisis en la investigación. Por otra parte, para la metodología 
cuantitativa está proyectado el uso de encuestas que determinarán la percepción del error inexcusable en los abogados litigantes, y cómo estos a su vez han sido afectados por el régimen disciplinario.

Sin embargo, en función de cumplir con el objetivo del actual artículo de investigación, se ha implementado una fase de investigación relacionada a la documental - bibliográfica, permitiendo a la investigadora generar una revisión del estado del arte del tema abordado, con lo cual se generó un análisis de documentos con la finalidad de presentar un primer avance investigativo, sustentando metodológicamente de la vertiente de Palella Stracuzzi y Martins Pestana (2012).

\section{RESULTADOS}

Como se mencionó anteriormente, en un Estado constitucional el conjunto de garantías destinadas a la efectividad del sistema judicial, el respeto a las partes en el proceso, las instituciones públicas y a proteger la libertad de los ciudadanos, constituye un núcleo esencial del sistema de derechos sobre el que se articula cualquier Estado constitucional (Zavala, 2010). Sin embargo, el error inexcusable incorporado en el régimen disciplinario judicial, establecido como causal gravísima de destitución, ha generado afectación a la estructura del sistema de justicia, a los operadores que imparten justicia y al ciudadano.

\section{Necesidades de un régimen disciplinario}

Es necesario entender que el juez es humano y, por ende, su actividad tiene que ser objeto de cuestionamientos, y aunque todos los jueces estén completamente de acuerdo en la decisión de uno en particular, esta, por tener efectos jurídicos que podrían involucrar consecuencias negativas a una de las partes procesales, la posibilidad de que se llegara a un consenso respecto a una determinada decisión judicial sería inexistente. De igual manera a este régimen disciplinario tampoco se le debe encasillar en la rigidez, ya que el constante control sobre las decisiones judiciales traería como consecuencia un derecho sin evolución, si ese fuese el caso, el adulterio o la homosexualidad seguirían siendo delitos. 
Martín del Burgo y Marchán (2001) indica que, en esa dirección, el juez 'humano' tiene una tarea amplia pues "tendrá que interpretar el sentido de la ley, llenar lagunas, resolver contradicciones de los textos legales, como las hay en las sentencias, en las que no faltarán [...] sentencias incómodas" (pág. 35) ante estas atribuciones que la ley le confiere genera una mayor probabilidad de inconformidad que podría ser calificada como "error".

La idea del error tampoco es solamente del juez, ya que la sistematización del derecho supone otra posibilidad para que existan equivocaciones, paradójicamente con la intención de salvaguardar la seguridad jurídica, "se pueden producir errores tanto en el procedimiento de sistematización como en el resultado obtenido por su intermedio" (Malem, 2009, pág. 24) por consiguiente la aplicación de un régimen disciplinario a los jueces por los errores de sistematización, es completamente absurdo e improcedente.

Es evidente que los sistemas modernos del Derecho reconocen al error como una realidad constante de la Justicia, y a pesar de la vía recursiva que el mismo sistema tiene, por ejemplo, la apelación, revisión o casación (en el caso del Ecuador) tratan de reducir al mínimo situaciones en donde la afectación a derechos sea grave, el régimen disciplinario, estaría enfocado a una última instancia para salvaguardar la integridad y transparencia del poder judicial.

\section{Un Error Inexcusable sin contenido}

En un sistema judicial donde los jueces y abogados están mayoritariamente apegados a una cultura jurídica normativista, la inclusión de un término jurídico sin definición no debería ser un problema si el sistema funcionara sin injerencia política, pues incluso en esos casos, el poder judicial toma a la doctrina como fuente para hacer frente a dichas lagunas. El argumento de la disociación es una de las herramientas de la argumentación jurídica que es compatible con cualquier sistema del Derecho, siendo así, se establecería una definición de 'error excusable' para entender al error inexcusable, consecuentemente, la definición del error inexcusable quedaría fijada para los jueces por la doctrina, e incluso la jurisprudencia también 
Iustitia Socialis. Revista Arbitrada de Ciencias Jurídicas.

Año IV. Vol. IV. N 1. Edición Especial 2019

Hecho el depósito de Ley: FA2016000064 ISSN: 2542-3371

FUNDACIÓN KOINONIA (F.K). Santa Ana de Coro, Venezuela

María Yokir Reyna Zambrano

podría darle una definición, reduciendo el riesgo a la arbitrariedad por falta de contenido de una norma.

No obstante, las problemáticas derivadas de esta causal de destitución no se originan necesariamente por dichos vacíos, sino más bien por los conflictos de interés que se presentaron durante el período analizado (2011 - 2017). El Pleno del Consejo de Participación Ciudadana Transitorio (2018) (en adelante PCPCCS - T) en la evaluación realizada al Consejo de la Judicatura, determinó que "la mayoría de los miembros del Consejo de Participación Ciudadana y Control Social cesado, no cumplían con las garantías de independencia y objetividad que debían acreditar de conformidad al artículo $232^{1}$ de la Constitución" (pág. 22). Dicho Consejo es el encargado del proceso de selección y designación de los miembros del Consejo de la Judicatura del Ecuador, por consiguiente, el mismo organismo, en ese mismo informe señala posteriormente que "la falta de independencia desde el origen del Consejo implica un grave indicio de que el procedimiento de designación de los vocales del Consejo de la Judicatura se encontró parcializado de raíz" (PCPSCCS T, 2018, pág. 25).

EI PCPCCS - T (2018) también concluye que existió una estrecha vinculación entre los vocales del Consejo de la Judicatura y la función ejecutiva; así como también entre ellos, por sus funciones desempeñadas en cargos anteriores (pág. 31). Tomando en cuenta que la Constitución y el COFJ le atribuye al Consejo de la Judicatura facultades disciplinarias es evidente que el régimen disciplinario coordinado por el organismo no constituye una herramienta para prevenir el error judicial. Siguiendo con el mismo informe, se concluye que el Consejo de la Judicatura manejó de forma arbitraria la información respecto a las irregularidades existentes en la función judicial (PCPCCS - T, pág. 50).

Siendo así, el papel que cumple el error inexcusable como causal de destitución sin contenido es la utilización de la misma para deshacerse de jueces que en sus sentencias hubieren fallado en contra de los intereses de altos funcionarios del Estado, o en su defecto de la voluntad de los mismos. Por ello, esta causal facilitó el

\footnotetext{
${ }^{1}$ No podrán ser funcionarias ni funcionarios ni miembros de organismos directivos de entidades que ejerzan la potestad estatal de control y regulación, quienes tengan intereses en las áreas que vayan a ser controladas o reguladas o representen a terceros que los tengan.
} 
camino a la manipulación de la justicia, el informe realizado por Pásara (2015) titulado "Independencia Judicial en la reforma de la justicia ecuatoriana" señala que el Error Inexcusable fue una de las dos causales más utilizadas por el Consejo de la Judicatura para llevar a cabo las destituciones desde las reformas del 2011. Según Páez (2013) "Ecuador ha estado inmerso en una estructura de poder que ha determinado que la justicia sea parte de un juego político" (pág. 115) y dicha afirmación, trae como resultado tales conceptos se tornen incompatibles, y por ende se consideren jurídicamente ineficaces.

\section{Sistema de Justicia y el conflicto de responsabilidades}

La seguridad jurídica, falta de independencia judicial y, por consiguiente, un servicio público de justicia se ha visto afectados. Adicionalmente, los procedimientos disciplinarios respecto a la destitución de jueces vulneran el derecho al debido proceso, y es que el COFJ establece que a quien le corresponde determinar la existencia del error inexcusable es a los jueces. Por lo tanto, las destituciones de dichos jueces por medio de resoluciones administrativas, sin un dictamen previo de un juez, como lo hizo el Consejo de la Judicatura, son ilegítimas por no seguir los procesos previstos por la ley y no respetar la vía recursiva. A pesar de aquello, los jueces simplemente fueron destituidos, afectando de manera directa al Sistema de la Carrera Judicial, garantiza la estabilidad y promoción de los magistrados siempre y cuando ellos no actúen contrario a la ley.

A la par, ante un sistema judicial en constante amenaza por el ejecutivo, son también los ciudadanos quienes se ven afectados, el informe realizado por Pásara (2015) identifica doce casos relevantes en donde se detectó injerencia política, adicionalmente, Human Rights Watch sostuvo que para inicios de 2014 ya 132 jueces habían sido destituidos por error inexcusable, y por fuentes obtenidas en el equipo del proyecto de investigación "La Destitución Administrativa de Jueces en el Estado Ecuatoriano por error inexcusable y su afectación a los derechos y garantías constitucionales, período 2011-2017", se ha contabilizado un número de 330 jueces hasta este primer trimestre de investigación, sumado a esto, están los jueces que 
para evitar ser destituidos dictaron sentencias atendiendo la voluntad de los actores políticos protagonistas de esta 'metida de mano' en la justicia ecuatoriana.

Este conflicto trajo como consecuencia una cadena de afectaciones, es así que la justicia contemplada en la Constitución y en COFJ como un servicio público, trae connotaciones aún más complicadas, empezando por las responsabilidades jurídicas, de la que se desprende la penal, civil y administrativa a la que nadie hizo frente, por tanto es incluso posible hablar de la responsabilidad social, ya que para el Ecuador, de manera generalizada se deja una percepción de una justicia 'no justa', que no opera de forma transparente, y que a pesar de tener "leyes buenas", dichas leyes simplemente no sirven.

Esta reflexión no es grave simplemente porque la gente lo diga, sino por la afectación que existe en términos conceptuales al Estado Constitucional pactado en el 2008 por los ecuatorianos, y ¿quién es el culpable de aquello? Se podría indicar que el inicio del conflicto empieza por la inclusión de una figura jurídica sin contenido en el ordenamiento jurídico, con poderes enormes en el sistema judicial disciplinario, esto es la destitución de jueces, por consiguiente, la responsabilidad sería del legislador, al omitir la definición del error inexcusable. Esta última afirmación indicaría que la definición del error inexcusable acabaría con el problema, sin embargo, decir esto es un error, pues al dirigirse a la historia ecuatoriana, en tiempos relativamente cercanos, donde no existía la causal "error inexcusable" en el ordenamiento jurídico, el sistema de justicia también carecía de 'una justicia justa' y con mayor evidencia se carecía de una designación de jueces que no tuvieren vínculos con el ejecutivo. Es así que, para el 2004 por ejemplo el ejecutivo destituiría a todos los jueces de Corte Nacional para colocar a jueces que le sirvieran al presidente, por lo que este conflicto no es nuevo, y tampoco es el único de esta clase acontecido en el país.

Las afectaciones por ende, son numerosas, y los casos en los que el Estado ha asumido su responsabilidad por la inadecuada administración de justicia y violación al derecho de tutela judicial efectiva son pocas, a tal punto que se los puede contabilizar, lo que no significa que las afectaciones sean solo esas, ya que mayoritariamente en el Ecuador, las personas por la situación económica en las que 
de manera generalizada se encuentran (esto es un estrato económico bajo con condiciones de vida regulares) están impedidas de acceder a los medios que le permitirían hacer que el Estado asuma la responsabilidad.

De la misma manera en las situaciones en donde el Estado le ha tocado asumir responsabilidades, este no hace uso del derecho de repetición, por lo que al final, por la arbitrariedad cometida por un alto funcionario quien termina pagando los errores son todos los ciudadanos. En situaciones económicas el Estado y los ciudadanos también se ven afectados por el constante despido de los jueces, ya que las inversiones realizadas por el gobierno en la Escuela de la Función Judicial terminan siendo un gasto donde el Estado nunca recupera lo invertido ante esta ola de destituciones injustificadas e ilegítimas.

Adicionalmente la Carrera Judicial, y por ende todas las actividades que realizan se ven limitadas, la calidad de las sentencias reducida, y el poder coercitivo que debe ejercer la justicia también. Por lo tanto, se estaría hablando de un Estado en donde sus entidades públicas simplemente no funcionan, y a la par este es un consecuente para que los problemas sociales continúen y se agudicen ante la falta de acción.

Es así que ven involucrados todos los actores que forman parte del Estado, los ciudadanos que están enmarcados en una democracia participativa de la que se entiende se crea para que sean ellos los encargados de velar por los intereses de los mismos de forma directa. Resulta curioso, pero necesario señalar que es precisamente el mismo pueblo, quien mediante consulta popular realizada en el año 2011 decide poner en manos del ejecutivo el control de la justicia, por medio de la pregunta $4^{2}$ dando como resultado una mayoría que estuvo de acuerdo. Dicha pregunta significaba la designación de un miembro para el Consejo de la Judicatura a cargo presidente, a la par, uno del parlamento, que en ese tiempo estaba en su mayoría llena del partido político a fin al presidente, y finalmente otro que pertenecería al quinto poder, tan criticado por considerarse también a fin del gobierno de ese entonces.

\footnotetext{
2 ¿Está usted de acuerdo en sustituir el actual pleno de la Judicatura por el Consejo de la Judicatura de Transición, conformado por tres miembros, uno por la Función Ejecutiva, otro por el Poder Legislativo y otro por la Función de Transparencia y Control Social, para que, en un plazo improrrogable de 18 meses, ejerza las competencias del Consejo de la Judicatura y reestructure la Función Judicial, como establece el anexo 4 ?
} 


\section{CONCLUSIONES}

Si bien es cierto cada nación evoluciona e involucra de manera diferenciada sus problemáticas sociales, es precisamente la tarea de las funciones públicas la que torna eficaz el propósito bajo el cual hubieren sido concebidas, ello en lo que respecta al Ecuador, la función judicial deja un vacío que apenas empieza a llenar, por consiguiente deja también este vacío en relación a la construcción del Estado Democrático y Constitucional de Derechos que la Constitución de la República del Ecuador inserta para con el país en el 2008.

Albert Camus alguna vez advirtió que si el hombre fallaba en la conciliación de la Justicia, este fallaría en todo, por ende, no es sorpresivo dirigirse al pasado y comprender, que las acciones realizadas por los antecesores ha dejado un legado diferente a la admirada herencia dada por dirigentes del poder judicial como John Marshall en los Estados Unidos, que dentro de su actividad jurisdiccional establece de manera clara y objetiva las facultades del organismo dentro de la construcción de un Estado de Derecho.

Las grandes revoluciones, y en especial la Revolución Francesa y la Revolución de las Trece Colonias han dejado de legado una concepción diferente a la expuesta por los anteriores feudalismo y esclavismo, y han ayudado al establecimiento del Estado como un supra organismo dedicado al servicio de sus ciudadanos. Ecuador frente a todos estos acontecimientos no ha contribuido a esta concepción. Pues como se ha visto, es aún el Estado quien no se abstiene de respetar los derechos que el mismo otorga, a cambio de las libertades que los ciudadanos si ceden al mismo, elementos constitutivos de un Estado de Derecho.

Sin duda alguna el Estado ha provocado un perjuicio a sí mismo, debilitando por medio del ejecutivo y de la democracia participativa la institucionalidad del mismo. Haciendo que las funciones bajo las cuales se creó el poder judicial por la doctrina francesa sean simplemente fantasía de los libros enmarcados en la utopía literaria, siguiendo las palabras de Sigmund Freud, el primer requisito de la civilización es la justicia, por lo que no se puede esperar una civilización completa si hace falta una justicia que responda a parámetros imparciales, alejados de la injerencia política y 
los conflictos de interés, en otras palabras una justicia imparcial que vele aplicando los principios y valores jurídicos sin mirar banderas de partidos.

Finalmente, respecto a la eliminación o no del error inexcusable como causal de destitución aplicable a los jueces, se concluye que el problema no es realmente la falta de contenido, sino más bien la utilización de este término jurídico que el ejecutivo le dio durante los años 2011 hasta el 2017 y la falta de eficacia que provocó el mismo gobierno para las leyes que habían sido creadas por el legislativo. Eliminarla no es tampoco una mala opción, pero si la creación de otras figuras que violenten la independencia del poder judicial, o en su defecto la acción ilegítima del ejecutivo sobre la función judicial, como fue el caso de la Pichi Corte en el 2004. Por otro lado, la definición de la figura legal en el ordenamiento jurídico no es tampoco una opción que no deba ser tomada en cuenta, pero en el caso de que se pretenda esto, se debe procurar evitar inmiscuirse en hacer un control respecto a una atribución que solo es designada a la función judicial jurisdiccional.

\section{REFERENCIAS CONSULTADAS}

1. Asamblea Nacional del Ecuador. (2011). Código Orgánico de la Función Judicial. Quito: Registro Oficial Suplemento 544.

2. Malem, J. (2009). El Error Judicial. En J. Malem, J. Ezquiaga, \& P. Ibáñez, El error judicial. La formación de los jueces (págs. 11-93). Madrid: Fundación Coloquio Jurídico Europeo.

3. Martín del Burgo y Marchán, Á. (2001). La Justicia como un problema. El juez como administrador del Derecho. Barcelona: Editorial Bosch S.A.

4. Páez, A. (2013). La metida de mano en la justicia. Quito: Paradiso Editores.

5. Pásara, L. (2015). Independencia Judicial en la Reforma de la Justicia Ecuatoriana.

6. Washington: Fundación para el Debido Proceso; Centro de Estudios de Derecho, Justicia y Sociedad; Instituto de Defensa Legal.

7. Palella Stracuzzi, S. \& Martins Pestana, F. (2012). Metodología de la investigación cuantitativa. Fondo editorial de la Universidad Pedagógica Libertador. Caracas, Venezuela. 
Iustitia Socialis. Revista Arbitrada de Ciencias Jurídicas.

Año IV. Vol. IV. N 1 . Edición Especial 2019

Hecho el depósito de Ley: FA2016000064 ISSN: $2542-3371$

FUNDACIÓN KOINONIA (F.K). Santa Ana de Coro, Venezuela

María Yokir Reyna Zambrano

8. Pleno del Consejo de Participación Ciudadana y Control Social Transitorio. (2018). Resolución PLE-CPCCS-T-O-037-04-06-2018. Quito.

9. Zaffaroni, R. (1992). Dimensión política de un poder judicial democrático. Quito: Corporación Latinoamericana para el Desarrollo.

10.Zavala, J. (2010). Derecho Constitucional, Neoconstitucionalismo y Argumentación Jurídica. Guayaquil: Edilex.

\section{REFERENCES CONSULTED}

1. National Assembly of Ecuador. (2011) Organic Code of the Judicial Function. Quito: Official Registry Supplement 544.

2. Malem, J. (2009). The Judicial Error In J. Malem, J. Ezquiaga, and P. Ibáñez, The judicial error. The formation of the judges (pp. 11-93). Madrid: European Legal Colloquium Foundation.

3. Martín del Burgo and Marchán, Á. (2001) Justice as a problem. The judge as administrator of the Law. Barcelona: Editorial Bosch S.A.

4. Páez, A. (2013). The hand in justice. Quito: Paradiso Editores.

5. Pásara, L. (2015). Judicial Independence in the Ecuadorian Justice Reform.

6. Washington: Due Process Foundation; Center for Law, Justice and Society Studies; Institute of Legal Defense.

7. Palella Stracuzzi, S. and Martins Pestana, F. (2012). Quantitative research methodology. Editorial Fund of the Libertador Pedagogical University. Caracas Venezuela.

8. Plenary session of the Citizen Participation and Temporary Social Control Council. (2018) Resolution PLE-CPCCS-T-O-037-04-06-2018. Quito

9. Zaffaroni, R. (1992). Political dimension of a democratic judiciary. Quito: Latin American Development Corporation.

10.Zavala, J. (2010). Constitutional Law, Neoconstitutionalism and Legal Argumentation. Guayaquil: Edilex. 